\title{
Estudio clínico de conciliación de la medicación al ingreso hospitala- rio en pacientes de las áreas de medicina interna y cirugía
}

\author{
Clinical study of medication reconciliation at hospital admission with patients in the \\ areas of internal medicine and surgery
}

José Antonio Mármol-Rodríguez ${ }^{1} \mathrm{M}^{\mathrm{a}}$ del Pilar Rodríguez-Casal ${ }^{2}$

1. XEFAR - Departamento Técnico Farmacéutico

2. Hospital SAS de Jerez - Unidad de Quirófano

\section{Artículo Original \\ Original Article \\ Correspondencia \\ Correspondence \\ D. José Antonio Mármol Rodríguez \\ Calle Arquitecto José Vargas, 2 \\ 11408 Jerez de la Frontera - Cádiz \\ T (34) 956150500 \\ marmol@jerez.es \\ Conflicto de interés \\ Competing interest \\ Los autores declaran que no existe} conflicto de interés.

Received: 30.12 .2014 Accepted: 10.05.2015

\section{cc) creative} LICENSE 3.0 UNPORTED.

\section{RESUMEN}

Objetivos. Examinar el grado de complejidad farmacoterapéutico en pacientes crónicos ingresados. Analizar la conciliación de la medicación al ingreso hospitalario. Detectar el número, naturaleza e impacto de los errores de conciliación. Analizar el protocolo de conciliación vigente y la aportación del farmacéutico al mismo.

Material y Métodos. Se analizó la medicación de pacientes de medicina interna y cirugía durante un mes en una clínica privada. La historia farmacoterapéutica se obtuvo a partir de la historia clínica y los registros de dispensación de medicamentos a las 48 horas post-ingreso. Se evaluaron las discrepancias de medicación, los errores asociados a ellas y la calidad del protocolo de conciliación.

Resultados. Fueron incluidos 60 pacientes con $62,5 \pm 17,2$ años de edad y con 2,7 $\pm 1,9$ enfermedades crónicas por paciente. El número de medicamentos habituales y hospitalarios por paciente fue $5,4 \pm 2,8$ y 6,0 $\pm 2,9$ respectivamente. Se observaron $2,6 \pm 2,5$ discrepancias no justificadas por paciente y éstas representaron el 33,5\%. El 49,3\% de los errores presentaron daño potencial asociado y 1,3 $\pm 1,9$ alcanzaron a cada paciente. La calidad del protocolo aumenta hasta el 94 \% con la intervención del farmacéutico.

Conclusiones. La farmacoterapia de los pacientes crónicos ingresados es compleja en el ámbito comunitario y hospitalario. Existe un número considerable de discrepancias no justificadas asociadas a errores graves al ingreso hospitalario. Los protocolos de conciliación deben ser revisados para alcanzar en la práctica una reducción de los errores de medicación y el farmacéutico debe tomar un papel activo en ellos.

Palabras clave: Conciliación de la Medicación. Errores de Medicación. Protocolo Clínico. Farmacia Asistencial.

\section{ABSTRACT}

Objectives. Examine the degree of complexity of pharmacotherapy in chronic patients. Analyze the continuity of medication at hospital admission. Detect the number, nature and impact of medication errors. Evaluate the hospital reconciliation protocol and analyze the contribution of the pharmacist.

Methodology. Patients in the areas of internal medicine and surgery were selected for a month. Sources consulted to elaborate the medication history were the medical history and listings of dispensing drugs in unit doses. 48 hours after admission, medication discrepancies 
and medication errors associated with them were evaluated. The current protocol was examined too.

Results. A total of 60 patients were included with an average age of $62.5 \pm 17.2$ years and $2.7 \pm 1.9$ per patient chronic diseases. The number of regular medication and during admission per patient was $5.4 \pm 2.8$ and $6.0 \pm 2.9$ respectively. $33.5 \%$ of the discrepancies were not justified and the average of them per patient was $2.6 \pm 2.5 .49 .3 \%$ of the discrepancies showed potential harm. An average of $1.3 \pm 1.9$ discrepancies with potential associated harm reached each patient. The medication reconciliation protocol, with the contribution of the pharmacist, was qualified with 94 out of 100 points.

Conclusions. Pharmacotherapy of chronic patients is complex in community and hospital services. There is a high number of unjustified discrepancies at admission. Medication reconciliation protocols must be reviewed to obtain quality standards and reduce medication errors. The pharmacist should collaborate in the protocol.

Keywords: Medication Reconciliation. Medication Errors. Clinical Protocol. Pharmaceutical Care.

\section{INTRODUCCIÓN}

La conciliación de la medicación ha sido definida como el proceso formal consistente en comparar al completo la medicación habitual del paciente con la medicación prescrita después de una transición asistencial ${ }^{1}$.Toda discrepancia entre ambas historias farmacoterapéuticas que no esté justificada por la situación clínica del paciente se considera un error de conciliación ${ }^{2}$. Este error requiere un análisis para valorar su gravedad y estrategias de intervención para comunicar y resolver interprofesionalmente los problemas reales y potenciales derivados de un uso inadecuado de la medicación.

La medicación es la primera causa de acontecimientos adversos que ponen en peligro la seguridad del paciente en el ámbito comunitario y hospitalario ${ }^{3,4}$. En este sentido, el proceso de conciliación es una de las estrategias para asegurar la continuidad e idoneidad del tratamiento en las diferentes estructuras de atención sanitaria como determina la legislación vigente ${ }^{5,6}$. Con él se garantiza al paciente recibir la medicación adecuada a sus necesidades clínicas en condiciones de óptima eficacia y seguridad ${ }^{7}$, y con ello, se reducen significativamente los errores de medicación ${ }^{8}$.

Entre las causas de los errores de conciliación se encuentran las enfermedades concomitantes, la polimedicación, la ausencia de registros unificados de salud, el tipo de unidades de atención, la transición considerada, la adaptación de la medicación habitual a la guía farmacoterapéutica y las características de la estancia hospitalaria9.

Existen artículos y revisiones que recogen cerca de treinta trabajos de investigación sobre conciliación de la medicación publicados hasta el momento 9,10. La mayoría han sido realizados en hospitales, han estudiado la medicación al ingreso y de ellos se concluye que entre 2 y 8 de cada 10 pacientes han sufrido errores de conciliación.

Por todo ello, desde hace ya una década, diferentes organizaciones vienen indicando la necesidad de incluir objetivos y protocolos concretos en materia de conciliación de la medicación para mejorar la seguridad de los pacientes ${ }^{11,12}$. Esto ha hecho que, para la acreditación de la calidad en unidades y centros, estos protocolos sean de obligatoria implantación.

El objetivo del presente estudio es analizar actualmente la adecuación de la continuidad del tratamiento de los pacientes ingresados, en un hospital provincial privado de especialidades médico-quirúrgicas, mediante los parámetros de conciliación de la medicación establecidos, examinar el grado de complejidad de la farmacoterapia, detectar el número, naturaleza e impacto de los errores de conciliación y evaluar la calidad del protocolo vigente en el centro conforme a las recomendaciones de conciliación ${ }^{13,14,15}$.

\section{MATERIAL Y MÉTODOS}

\section{Fuentes de información}

Los datos clínicos y de medicación se han extraído de las fuentes impresas y en soporte informático presentes en las áreas de farmacia y enfermería. Las fuentes consultadas han sido la historia clínica y el registro de dispensación de dosis unitarias a las 48 horas tras el ingreso. El tiempo de reclutamiento de pacientes ha sido de un mes completo.

\section{Criterios de inclusión}

Se han seleccionado pacientes crónicos de medicina interna y cirugía para proveer, presumiblemente, al estudio de pacientes que hayan ingresado con un tratamiento crónico o algún otro tratamiento previo. El tiempo de muestreo de datos de medicación hospitalaria se ha establecido con tiempo suficiente para poder valorar la respuesta del protocolo de conciliación establecido.

\section{Parámetros de estudio en pacientes}

Los datos obtenidos han sido la edad, sexo, medicación previa, medicación hospitalaria, alergias y hábitos tóxicos. Todos ellos han sido incorporados una ficha modelo preparada a tal efecto que mantiene la privacidad del paciente mediante la disociación de sus datos identificativos. 
Las discrepancias no justificadas de medicación y el nivel de gravedad de sus errores asociados han sido evaluadas conforme a los parámetros establecidos por la bibliografía de referencia nacional e internacional ${ }^{13,14}$

Las discrepancias de interacción han sido doblemente evaluadas con las aplicaciones informáticas españolas BOT Plus 2.0 y Vademecum.es. Sólo se han contabilizado las interacciones denominadas «graves» (BOT Plus 2.0) o «potencialmente graves» (Vademecum.es) otorgándoles una categoría de error del tipo «D» dado que no se ha tenido constancia de manifestación clínica durante el período de estudio.

Las discrepancias correspondientes a los subtipos «diferente medicamento» (sustitución no justificada) y «medicamento no disponible en el hospital» se han establecido conforme a la guía farmacoterapéutica vigente de la clínica en el período de estudio.

\section{Parámetros de estudio en el protocolo de conciliación}

El protocolo de conciliación de la medicación al ingreso hospitalario ha sido evaluado conforme a las recomendaciones de la Junta de Andalucía (JJAA) adaptadas del cuestionario elaborado a tal efecto por el Instituto para el Uso Seguro de los Medicamentos (ISMP) de aplicación nacional e internacional ${ }^{12,15}$.

Junto a cada una de las recomendaciones, se incluye una puntuación máxima la cual se ha asignado al protocolo, en mayor o menor medida, en función del grado de adecuación a los ítems de la guía. La valoración y puntuación de cada recomendación se ha efectuado siguiendo los criterios detallados en el cuestionario de autoevaluación de la seguridad del sistema de utilización de los medicamentos de ISMP-España ${ }^{12}$. Existen tres ítems JJAA que no encuentran correspondencia con ISMP. A cada uno de ellos se le ha otorgado un máximo de 2 puntos para que el total de puntuación del baremo se exprese sobre 100 puntos. Para estas tres recomendaciones se ha valorado su presencia (2 puntos) o su ausencia ( 0 puntos) en el protocolo del centro.

Las recomendaciones se han divido en tres categorías:

1. Recomendaciones que atañen a la elaboración del protocolo de conciliación desde la farmacia.

2. Recomendaciones que pueden incluirse en el protocolo de conciliación desde la farmacia

3. Recomendaciones fuera del alcance del farmacéutico en el presente protocolo.

Se ha reelaborado y valorado nuevamente el protocolo atendiendo a las categorías primera y segunda.

\section{RESULTADOS}

La edad media de los pacientes incluidos ha sido de $62,5 \pm$ 17,2 años. La distribución de pacientes por sexos ha resultado ser de 51,6 \% varones y $48,3 \%$ mujeres.

Se observaron 2,7 $\pm 1,9$ enfermedades crónicas por paciente. Estas patologías, las motivadoras del ingreso y las sobrevenidas durante el transcurso de la estancia hospitalaria, han sido tratadas con 5,4 $\pm 2,8$ (preingreso) y 6,0 $\pm 2,9$ (ingreso) medicamentos respectivamente.

La historia clínica ha ofrecido información completa de la historia farmacoterapéutica previa al ingreso en el 28,6 \% de los pacientes, incompleta en el 50,0 \% de los casos y ha estado ausente en el 21,4 \% de los pacientes. En las historias incompletas, faltaban por consignarse los datos de dosis (3,6 de cada 5), forma farmacéutica/vía de administración (4,6 de cada 5) y pauta posológica (4,0 de cada 5). Sólo 2,6 de cada 5 medicamentos había sido consignado por principio activo.

Los datos de alergias o reacciones adversas a medicamentos (RAMs) estaban presentes en el 89,3\% de los casos. De ellos, el 22,0 \% de los pacientes habían presentado episodios de RAMs. Los datos de hábitos tóxicos que influyen en la eficacia y seguridad del tratamiento estaban presentes en el 39,3 \% de los casos. De ellos, el 68,2 \% de los pacientes presentaba hábitos tóxicos, fundamentalmente alcohol, tabaco y dependencias a medicamentos.

Las discrepancias justificadas se han cuantificado en 4,5 \pm 2,8 por paciente frente a las discrepancias no justificadas que han resultado ser 2,6 $\pm 2,5$ por paciente. En las Tablas 1 y 2 se presentan algunos resultados representativos de las discrepancias injustificadas detectadas en el presente estudio y de la valoración posterior del error asociado a ellas. En las Tablas 3 y 4 se ofrecen detalladamente los datos de medicamentos conciliados y las diferentes tipologías de discrepancias detectadas.

La gravedad de los errores asociados a las discrepancias no justificadas de medicación se ha reflejado de manera pormenorizada en la Tabla 5. Se han encontrado 0,9 $\pm 0,5$ errores que «requieren monitorización y/o intervención» y una media de 1,3 $\pm 1,9$ «daños potenciales» por paciente.

La calidad del protocolo de conciliación de la medicación ha sido valorada en 52 puntos sobre 100. Tras su revisión y reelaboración, se ha observado que, incluir los ítems en los que el farmacéutico toma parte asistencial, incrementa notablemente la calidad de este procedimiento clínico hasta 94 puntos sobre 100. En la Tabla 6 se detallan las puntuaciones del protocolo previo y del protocolo reelaborado. 
Tabla 1. Análisis de casos clínicos de discrepancias injustificadas de medicación.

\begin{tabular}{|c|c|c|c|}
\hline $\begin{array}{l}\text { Tipo de discrepancia no justi- } \\
\text { ficada }^{13}\end{array}$ & Características del paciente & Medicación previa & Medicación hospitalaria \\
\hline Omisión de medicamento & $\begin{array}{l}\text { Afectado de neoplasia con } \\
\text { manifestación hiperuricémica } \\
\text { moderada. }\end{array}$ & $\begin{array}{l}\text { Alopurinol } 300 \text { mg Comp. } \\
\text { VO } 1 / 24 \mathrm{~h}\end{array}$ & $\begin{array}{l}\text { Sin tratamiento del mismo } \\
\text { grupo o equivalente }\end{array}$ \\
\hline Inicio de medicación & $\begin{array}{l}\text { Afectado de constipación de un } \\
\text { solo día de evolución, hidrata- } \\
\text { do, sin enfermedad digestiva, } \\
\text { cirugía, endoscopia o radiología } \\
\text { próxima. }\end{array}$ & Sin medicación previa & $\begin{array}{l}\text { Fosfato de Sodio } 250 \mathrm{~mL} \\
\text { Sol. VR } 1 / 24 \mathrm{~h}\end{array}$ \\
\hline $\begin{array}{l}\text { Cambio de dosis, pauta o vía de } \\
\text { administración }\end{array}$ & $\begin{array}{l}\text { Afectado de infección urinaria. } \\
\text { En tratamiento de manteni- } \\
\text { miento y reducción de dosis } \\
\text { progresiva con antiarrítmicos. }\end{array}$ & $\begin{array}{l}\text { Amiodarona } 200 \mathrm{mg} \\
\text { Comp. VO } 1 / 48 \mathrm{~h}\end{array}$ & $\begin{array}{l}\text { Amiodarona } 200 \mathrm{mg} \\
\text { Comp. VO } 1 / 24 \mathrm{~h}\end{array}$ \\
\hline Diferente medicamento & $\begin{array}{l}\text { Anticoagulado por riesgo de } \\
\text { tromboembolismo no quirúr- } \\
\text { gico. }\end{array}$ & $\begin{array}{l}\text { Enoxaparina } 6000 \text { UI Iny. } \\
\text { VSc } 1 / 24 \mathrm{~h}\end{array}$ & $\begin{array}{l}\text { Bemiparina } 3500 \text { UI Iny. } \\
\text { VSc } 1 / 24 \text { h }\end{array}$ \\
\hline Duplicidad & $\begin{array}{l}\text { Intervenido que requiere seda- } \\
\text { ción continua postquirúrgica. }\end{array}$ & $\begin{array}{l}\text { Midazolam } 15 \text { mg Iny. IV } \\
1 / 6 \mathrm{~h}\end{array}$ & $\begin{array}{l}\text { Midazolam } 15 \text { mg Iny. IV } \\
1 / 6 \mathrm{~h}(\mathrm{x} 2)\end{array}$ \\
\hline Interacción & $\begin{array}{l}\text { Anticoagulado en tratamiento } \\
\text { de dolor leve-moderado post- } \\
\text { quirúrgico. }\end{array}$ & $\begin{array}{l}\text { Ácido Acetilsalicílico } 500 \\
\text { mg Comp. VO 1/24 h }\end{array}$ & $\begin{array}{l}\text { Ácido Acetilsalicílico } 500 \\
\text { mg Comp. VO } 1 / 24 \text { h } \\
\text { Bemiparina } 3500 \text { UI Iny. } \\
\text { VSc. } 1 / 24 \text { h } \\
\text { Dipirona } 2 \text { g Iny. IV } 1 / 8 \text { h } \\
\text { Dexketoprofeno } 50 \text { mg Iny. } \\
\text { IV } 1 / 8 \text { h }\end{array}$ \\
\hline $\begin{array}{l}\text { Medicamento no disponible en } \\
\text { el hospital }\end{array}$ & $\begin{array}{l}\text { Diagnosticado de diabetes } \\
\text { mellitus tipo II. }\end{array}$ & $\begin{array}{l}\text { Insulina lispro } 100 \mathrm{U} \text { Iny. } \\
\text { VSc } 1 / \text { Ingesta }\end{array}$ & $\begin{array}{l}\text { Insulina glargina } 100 \mathrm{UI} \\
\text { Iny. VSc } 1 / 12 \mathrm{~h}\end{array}$ \\
\hline
\end{tabular}

$\mathrm{VO}=$ vía oral, $\mathrm{VR}=$ =ía rectal, $\mathrm{VSc}=$ =vía subcutánea, IV=vía intravenosa, Comp.= comprimido, Cáps.= cápsula, Sol.=solución, Iny. $=$ inyectable.

Tabla 2. Casos clínicos con valoración del error asociado a discrepancias injustificadas de medicación.

\begin{tabular}{|l|l|l|l|l|}
\hline $\begin{array}{l}\text { Categoría del daño asocia- } \\
\mathbf{d o}^{\mathbf{1 3 , 1 4}}\end{array}$ & $\begin{array}{l}\text { Tipo de discrepan- } \\
\text { cia no justificada }\end{array}$ & $\begin{array}{l}\text { Características de } \\
\text { paciente }\end{array}$ & Medicación previa & $\begin{array}{l}\text { Medicación hospi- } \\
\text { talaria }\end{array}$ \\
\hline Sin daño potencial (A-C) & $\begin{array}{l}\text { Cambio de dosis, vía } \\
\text { o frecuencia. }\end{array}$ & $\begin{array}{l}\text { Paciente mayor con } \\
\text { ansiedad e insomnio } \\
\text { crónico controlado. }\end{array}$ & $\begin{array}{l}\text { Alprazolam } 0,25 \mathrm{mg} \\
\text { Comp. VO } 1 / 24 \mathrm{~h}\end{array}$ & $\begin{array}{l}\text { Alprazolam } 0,5 \mathrm{mg} \\
\text { Comp. VO } 1 / 24 \mathrm{~h}\end{array}$ \\
\hline $\begin{array}{l}\text { Daño que requiere monito- } \\
\text { rización o intervención para } \\
\text { evitarlo (D) }\end{array}$ & $\begin{array}{l}\text { Omisión de medi- } \\
\text { cación. Prescripción } \\
\text { inadecuada. }\end{array}$ & $\begin{array}{l}\text { Afectado de dolor on- } \\
\text { cológico moderado- } \\
\text { severo. }\end{array}$ & $\begin{array}{l}\text { Tramadol } 100 \mathrm{mg} / \mathrm{mL} \\
\text { Sol. VO } 100 \mathrm{mg} / 8 \mathrm{~h} \\
\text { Dipirona } 575 \mathrm{mg} \\
\text { Cáps. VO } 1 / 8 \mathrm{~h}\end{array}$ & $\begin{array}{l}\text { Dipirona } 575 \mathrm{mg} \\
\text { Cáps. VO } 1 / 8 \mathrm{~h} \\
\text { Paracetamol } 1 \mathrm{~g} \\
\text { Comp. VO } 1 / 8 \mathrm{~h}\end{array}$ \\
\hline Daño potencial (E-I) & $\begin{array}{l}\text { Omisión de medi- } \\
\text { cación. }\end{array}$ & $\begin{array}{l}\text { Anticoagulado sin } \\
\text { cirugía próxima, he- } \\
\text { morragia, o condicio- } \\
\text { nes similares. }\end{array}$ & $\begin{array}{l}\text { Acenocumarol } 4 \mathrm{mg} \\
\text { Comp. VO } 0,5 / 24 \mathrm{~h}\end{array}$ & $\begin{array}{l}\text { Sin tratamiento } \\
\text { del mismo grupo o } \\
\text { equivalente. }\end{array}$ \\
\hline
\end{tabular}

VO=vía oral, VR=vía rectal, VSc=vía subcutánea, IV=vía intravenosa, Comp.= comprimido, Cáps.= cápsula, Sol.=solución, Iny. $=$ inyectable.

Tabla 3. Distribución de medicamentos conforme a su conciliación.

\begin{tabular}{|l|l|l|}
\hline Discrepancias de conciliación & Medicamentos $(\mathbf{n}=\mathbf{4 4 2})$ & $\%$ \\
\hline Sin discrepancia & 39 & 8,8 \\
\hline Discrepancias justificadas & 255 & 57,7 \\
\hline Discrepancias no justificadas & 148 & 33,5 \\
\hline
\end{tabular}


Tabla 4. Distribución de medicamentos por tipos de discrepancias no justificadas.

\begin{tabular}{|l|l|l|}
\hline Discrepancia injustificada $^{13}$ & Medicamentos (n=148) & $\%$ \\
\hline Omisión & 70 & 47,3 \\
\hline Inicio de medicación & 8 & 5,4 \\
\hline Cambio de dosis/vía/pauta & 5 & 3,4 \\
\hline Diferente medicamento & 8 & 5,4 \\
\hline Duplicidad & 3 & 2,0 \\
\hline Interacción & 41 & 27,7 \\
\hline Medicación no disponible & 13 & 8,8 \\
\hline
\end{tabular}

Tabla 5. Distribución discrepancias no justificadas en función de su error asociado.

\begin{tabular}{|l|l|l|}
\hline Categoría de error* & Discrepancias $(\mathbf{n}=\mathbf{1 4 8})$ & $\%$ \\
\hline B & 7 & 4,7 \\
\hline C & 17 & 11,5 \\
\hline D & 51 & 34,5 \\
\hline E & 46 & 31,1 \\
\hline F & 27 & 18,2 \\
\hline
\end{tabular}

${ }^{*}$ ) Sin daño potencial: comprende las categorías A-C. Requieren monitorización o intervención para evitar el daño: incluye la categoría D. Daño potencial: abarca las categorías E-I.

$\mathrm{B}=$ Error que no alcanza al paciente; no causa daño.

$\mathrm{C}=$ Error que alcanza al paciente; no es probable que cause daño.

$\mathrm{D}=$ Error que alcanza al paciente; necesita monitorización y/o intervención para evitarse.

$\mathrm{E}=$ Error que hubiera causado daño temporal.

$\mathrm{F}=$ Error que hubiera causado daño que requiere hospitalización.

Tabla 6. Valoración del protocolo de conciliación de la medicación.

\begin{tabular}{|c|c|c|c|c|}
\hline Ítems y categoría asociada & $\begin{array}{l}\text { Puntuación máxima } \\
\text { ISMP }^{12}-\mathrm{JJAA}^{15}\end{array}$ & Valoración previa & $\begin{array}{l}\text { Valoración pos- } \\
\text { terior* }\end{array}$ & $\begin{array}{l}\text { Incremento pun- } \\
\text { tuación }\end{array}$ \\
\hline $\begin{array}{l}\text { Categoría A } \\
\text { Recomendaciones } \mathrm{n}^{\circ} \\
1,4,5,7,9,10,11,12 \text { y } 18\end{array}$ & 62 & 21 & 62 & 42 \\
\hline $\begin{array}{l}\text { Categoría B } \\
\text { Recomendaciones } n^{\circ} \\
2,3,13 \text { y } 14\end{array}$ & 26 & 20 & 21 & 0 \\
\hline $\begin{array}{l}\text { Categoría C } \\
\text { Recomendaciones } \mathrm{n}^{\circ} \\
6,8,15,16 \text { y } 17\end{array}$ & 12 & 12 & 12 & 0 \\
\hline Total & 100 & 52 & 94 & 42 \\
\hline
\end{tabular}

A=Atañe a la elaboración del protocolo de conciliación desde la farmacia.

$\mathrm{B}=$ Puede incluirse en el protocolo de conciliación.

C=Está fuera del alcance del farmacéutico en el presente protocolo de conciliación.

${ }^{*}$ ) Tras establecerse las recomendaciones de farmacia asistencial en el protocolo. 


\section{DISCUSIÓN}

La metodología utilizada y los resultados obtenidos por el presente estudio se encuentran en sintonía con la relación de estudios precedentes ${ }^{9,10}$. El farmacéutico, con ayuda del personal de enfermería, ha sido el profesional encargado de recoger los datos y valorar la conciliación de medicamentos en este estudio. Este mismo hecho ha supuesto en anteriores estudios que más del $70 \%$ de los pacientes presentaran conciliada su medicación a las 48 horas tras el ingreso ${ }^{16}$.

Destacan tres novedades metodológicas. La primera de ellas, es la elaboración de un formulario de recogida de datos (véase Anexo), frente al 72,0 \% de trabajos examinados en los que no se hace referencia expresa al uso de los mismos ${ }^{9,10}$. La segunda, es la utilización del registro de dispensación de dosis unitarias, documento exclusivo del área de farmacia, como fuente para la historia farmacoterapéutica. La tercera, es haber realizado un amplio análisis de discrepancias, haber establecido la gravedad de los errores asociados a ellas, y haber considerando también los datos de alergias y/o RAMs y de hábitos tóxicos estrechamente ligados a la seguridad y eficacia de los tratamientos.

La edad de la población de estudio abarca un intervalo considerable que representa gran parte de la población susceptible de un ingreso hospitalario. La población está distribuida homogéneamente por sexo.

El número de medicamentos empleados por el paciente en el ámbito extrahospitalario parece concordar con la media de enfermedades crónicas y su correspondiente desviación. Parece, por tanto, que en líneas generales, los pacientes tratan en número adecuado de medicamentos sus enfermedades crónicas en el ámbito comunitario. Cabe recordar aquí que cada enfermedad crónica es susceptible de controlarse con uno, dos o incluso varios medicamentos.

El número de medicamentos hospitalarios dispensados por el área de farmacia parece concordar también con el número de medicamentos prescritos en el ámbito comunitario. Por tanto, a priori parece que la medicación se encuentra bien conciliada en número en el ámbito hospitalario. Los datos de medias suavizan presumiblemente los errores por falta o aumento de medicación. Este número de discrepancias y la prescripción o desprescripción extraordinaria, que conlleva el manejo de las afecciones desencadenantes del ingreso en el hospital, puede justificar la leve variación en la media del número de medicamentos al ingreso frente al número de medicamentos habituales por paciente.

El porcentaje de errores de conciliación al ingreso hospitalario y la media de estas discrepancias por paciente están en consonancia con estudios previos ${ }^{17,18}$. La variabilidad otorgada por la desviación calculada sugiere que la mayor o menor presencia de discrepancias viene determinada por el número total de medicamentos del paciente, de sus patologías presentes y de la variación del tratamiento en el hospital $^{19}$. Dedúzcase, por ejemplo, que un número elevado de prescripciones aumenta la probabilidad de interacciones y puede dificultar también el correcto análisis del total de prescripciones, lo que propicia las demás discrepancias contempladas.

Destaca el alto número de pacientes con información de medicación incompleta -datos de RAMs y/o hábitos tóxicos- teniendo en cuenta la especial atención que ha de prestarse a ellos en el paciente hospitalizado.

Los medicamentos correctamente conciliados han representado un aceptable porcentaje. Por el contrario, sigue existiendo un alto número de discrepancias injustificadas de medicación. Las más destacadas han sido las discrepancias por omisión e interacción. El primer dato se encuentra en línea con la mayoría de estudios efectuados que presentan el error de omisión como uno de los más comunes ${ }^{20,21}$. El segundo parece ser comprensible; cerca del $60 \%$ de los pacientes han sido tratados en el hospital con más de 5 medicamentos. El resto de discrepancias han obtenido tanto individualmente como en conjunto valores algo menores pero con gran significancia en clínica por los errores asociados a ellas.

El promedio y desviación de los datos de daños graves permite demostrar un mayor alcance y prevalencia en los pacientes. Las desviaciones obtenidas en los promedios calculados para cada tipo y grado de error son mucho menores que las obtenidas en otros apartados del presente trabajo, lo que reduce la tasa de variación interindividual con respecto al promedio de errores graves por paciente. Los porcentajes de errores graves (daño potencial) parecen estar de acuerdo también con estudios previos que los sitúan en torno al $50 \%$.

Finalmente, la puntuación obtenida por el protocolo previo parece corresponderse con la tasa de errores detectados. La mejora del protocolo en los puntos de intervención farmacéutica permiten mejorar ampliamente su calidad y puntuación, lo que presupondrá una reducción significativa de los errores reportados.

Existen limitaciones metodológicas del presente trabajo. Por ejemplo, no se ha completado la historia farmacoterapéutica previa mediante entrevista con el paciente, la historia clínica contempla poco los medicamentos que no requieren receta y el registro de dispensaciones unitarias puede no corresponderse con la medicación que es finalmente administrada. Puede ser probable también, que el 
número de sujetos, la franja de edad y el área de ingreso de los pacientes estudiados no representen de un modo completo las posibles discrepancias y errores asociados del resto y conjunto de la población hospitalizada.

\section{CONCLUSIONES}

La mayoría de pacientes ingresados en la clínica presenta un número notable de enfermedades crónicas. El número de medicamentos asociados a ellas y el número de medicamentos añadidos al ingreso hospitalario hacen compleja la conciliación de los medicamentos.

En la actualidad, sigue existiendo un elevado número de discrepancias injustificadas de la medicación en la transición considerada en el presente estudio. A su vez, destaca que la magnitud del error asociado a ellas es grave, o potencialmente grave, en la mitad de los casos. Destaca también que cada paciente es susceptible de recibir, al menos, un error grave de medicación. La calidad mejorable del protocolo de conciliación sugiere los resultados obtenidos en los parámetros de conciliación.

El incremento en calidad del protocolo para la conciliación de medicamentos es notorio tras incluir activamente al farmacéutico en este procedimiento asistencial. Los protocolos deben ser revisados para asegurar su eficiencia y aplicabilidad, y deben coordinar la labor de los profesionales médicos, farmacéuticos y de enfermería responsables de la correcta continuidad interasistencial de la prescripción, dispensación y administración de los tratamientos farmacológicos.

\section{ANEXO}

Formulario de recogida de datos y análisis de conciliación de la medicación.

Datos del paciente

\begin{tabular}{|l|l|l|l|}
\hline No de Registro: & & Edad: & \\
\hline$N^{\circ}$ Enf. Crónicas: & & Sexo: & V $\square$ M \\
\hline RAMs/Alergias & Documentado: Sí $\square$ No & Eventos conocidos: Sí $\square$ No $\square$ \\
\hline Hábitos Tóxicos: & Documentado: Sí $\square$ No & Hábitos conocidos: Sí $\square$ No $\square$ \\
\hline
\end{tabular}

Datos de medicación

\begin{tabular}{|c|c|c|c|c|c|c|c|c|c|c|c|c|c|}
\hline \multicolumn{7}{|c|}{ Medicación preingreso } & \multicolumn{7}{|c|}{ Medicación durante el ingreso } \\
\hline Medicación & $\mathrm{D}$ & $\mathrm{P}$ & FF & V & Di & $\mathrm{E}$ & Medicación & $\mathrm{D}$ & $\mathrm{P}$ & FF & V & $\mathrm{Di}$ & E \\
\hline & & & & & & & & & & & & & \\
\hline & & & & & & & & & & & & & \\
\hline & & & & & & & & & & & & & \\
\hline & & & & & & & & & & & & & \\
\hline & & & & & & & & & & & & & \\
\hline & & & & & & & & & & & & & \\
\hline & & & & & & & & & & & & & \\
\hline & & & & & & & & & & & & & \\
\hline & & & & & & & & & & & & & \\
\hline & & & & & & & & & & & & & \\
\hline
\end{tabular}

\section{$\underline{\mathrm{D}=\text { Dosis }} \quad \underline{\mathrm{P}=\text { Pauta }} \quad \underline{\mathrm{FF}=\text { Forma Farmacéutica }}$}

\section{Di=Discrepancia}

1) Sin discrepancia.

2) Discrepancias justificadas por la situación clínica.

3) Discrepancia injustificada:
3a) Omisión
3b) Inicio de medicación
3c) Cambio de dosis/vía/pauta
3d) Diferente medicamento
3e) Duplicidad
3f) Interacción
3g) Medicación no disponible

E=Error asociado a discrepancias

A) No hay error, pero es posible que se produzca.

B) Error que no alcanza al paciente; no causa daño.

C) Error que alcanza al paciente pero no es probable que cause daño.

D) Error que alcanza al paciente y necesitaría monitorización y/o intervención para evitar el daño.

E) Error que hubiera causado daño temporal.

F) Error que hubiera causado daño y requeriría hospitalización o prolongación de la estancia.

G) Error que hubiera causado daño permanente.

H) Error que hubiera requerido soporte vital.

I) Error que hubiera resultado mortal. 


\section{REFERENCIAS}

1. Gleason KM1, Groszek JM, Sullivan C, Rooney D, Barnard C, Noskin GA. Reconciliation of discrepancies in medication histories and admission orders of newly hospitalized patients. Am J Health Syst Pharm. 2004;61: 1689-95.

2. Conciliación de la medicación. Información Farmacoterapéutica de la Comarca (INFAC). 2013;21(10):68-74.

3. Estudio sobre la seguridad de los pacientes en atención primaria de salud (APEAS). Ministerio de Sanidad y Consumo. Madrid; 2008.

4. Estudio nacional sobre los efectos adversos ligados a la hospitalización (ENEAS). Ministerio de Sanidad y Consumo. Madrid; 2005.

5. Ley $29 / 2006$, de 26 de julio, de garantías y uso racional de los medicamentos y productos sanitarios.

6. Ley 44/2003, de 21 de noviembre, de ordenación de las profesiones sanitarias.

7. The Rational Use of Drugs - Report of the Conference of Experts. Organización Mundial de la Salud. Nairobi; 1985. Disponible en: http://apps.who.int/medicinedocs/es/m/abstract/Js17054e/ (Sep 2014).

8. Varkey P, Resar RK. Medication reconciliation implementation in an academic center. Am J Med Qual. 2006;21:293-295.

9. Delgado O, Anoz L, Serrano A, Nicolás J. Conciliación de la medicación. MedClin (Barc). 2007;129(9):343-8.

10. Alfaro ER, Vega MD, Galván M, Nieto MD, Pérez C, Santos B. Metodología de conciliación del tratamiento farmacológico en pacientes pluripatológicos. Aten Primaria. 2014;46(2):8999.

11. Medication reconciliation handbook. Joint Commission on Accreditation of Healthcare Organizations. Oakbrook Terrace, IL: Joint Commission Resources; 2006.

12. Cuestionario de autoevaluación de la seguridad del sistema de utilización de los medicamentos en los hospitales. Instituto para el Uso Seguro de los Medicamentos. Delegación Española del Institute for Safe Medication Practices (ISMP). Disponible en: http://apps.ismp-espana.org/ficheros/cuestionario. pdf (Sep 2014)
13. Documento de consenso en terminología y clasificación de los programas de conciliación de la medicación. Sociedad Española de Farmacia Hospitalaria (SEFH). Editorial Mayo. Madrid; 2009.

14. Index for Categorizing Medication Errors. The National Coordinating Council for Medication Error Reporting and Prevention (NCC MERP). Disponible en: http://www.nccmerp.org/medErrorCatIndex.html (Sep 2014).

15. Buenas prácticas en la conciliación de la medicación en el ingreso, alta y transición interservicios. Observatorio para la seguridad del paciente. Agencia de Calidad Sanitaria de Andalucía. Junta de Andalucía. Sevilla; 2010.

16. Beckett RD, Crank CW, Wehmeyer A. Effectiveness and feasibility of pharmacist-led admission medication reconciliation for geriatric patients. J Pharm Pract. 2012;25:136-141.

17. Gizzi LA, Slain D, Hare JT, Sager R, Briggs F, Palmer CH. Assessment of a safety enhancement to the hospital medication reconciliation process for elderly patients. Am J GeriatrPharmacother. 2010;8:127-135.

18. Knez L, Suskovic S, Rezonja R, Laaksonen R, Mrhar A. The need for medication reconciliation: A cross-sectional observational study in adult patients. Respir Med. 2011;105:60-66.

19. Lubowski TJ, Cronin LM, Pavelka RW, Briscoe LA, Briceland LL, Hamilton RA, Effectiveness of a medication reconciliation project conducted by PharmD students. Am J Pharm Educ. 2007;71:94.

20. Kemp LO, Narula P, McPherson ML, Zuckerman I. Medication reconciliation in hospice: A pilot study. Am J HospPalliat Care. 2009;26:193-199.

21. Gleason KM, McDaniel MR, Feinglass J, Baker DW, Lindquist L, Liss L, et al.. Results of the Medications at Transitions and Clinical Handoffs (MATCH) study: An analysis of medication reconciliation errors and risk factors at hospital admission. J Gen Intern Med. 2010;25:441-447.

22. Pippins JR, Gandhi TK, Hamann C, Ndumele CD, Labonville SA, Diedrichsen EK, et al.. Classifying and predicting errors of inpatient medication reconciliation. J Gen Intern Med. 2008;23:1414-1422 